WS. Mutations in the Fas antigen in patients with multiple myeloma. Blood. 1997;90(11):4266-4270.

9. Tamiya S, Etoh K, Suzushima H, Takatsuki K, Matsuoka M. Mutation of CD95 (Fas/Apo-1) gene in adult T-cell leukemia cells. Blood. 1998; 91(10):3935-3942.

10. Beltinger C, Kurz E, Bohler T, Schrappe M, Ludwig WD, Debatin KM. CD95 (APO-1/Fas) mutations in childhood T-lineage acute lymphoblastic leukemia. Blood. 1998;91(10):3943-3951.

11. Fisher GH, et al. Dominant interfering Fas gene mutations impair apoptosis in a human autoimmune lymphoproliferative syndrome. Cell. 1995 ; 81(6):935-946.

12. Jackson CE, et al. Autoimmune lymphoproliferative syndrome with defective Fas: genotype influences penetrance. Am J Hum Genet. 1999; 64(4):1002-1014

13. Caminha I, et al. Using biomarkers to predict the presence of FAS mutations in patients with features of the autoimmune lymphoproliferative syndrome. J Allergy Clin Immunol. 2010; 125(4):946-949.

14. Bleesing JJ, et al. A composite picture of TcR alpha/ beta(+) CD4(-)CD8(-) T Cells (alpha/beta-DNTCs) in humans with autoimmune lymphoproliferative syndrome. Clin Immunol. 2002;104(1):21-30.

15. Landolfi MM, Van Houten N, Russell JQ, Scollay R, Parnes JR, Budd RC. CD2-CD4-CD8-lymph node T lymphocytes in MRL lpr/lpr mice are derived from a CD2+CD4+CD8+ thymic precursor. J Immunol. 1993;151(2):1086-1096.
16. Sobel ES, Kakkanaiah VN, Rapoport RG, Eisenberg RA, Cohen PL. The abnormal lpr double-negative T cell fails to proliferate in vivo. Clin Immunol Immunopathol. 1995;74(2):177-184.

17. Stranges PB, et al. Elimination of antigen-presenting cells and autoreactive $T$ cells by Fas contributes to prevention of autoimmunity. Immunity. 2007;26(5):629-641.

18. Izui S, Kelley VE, Masuda K, Yoshida H, Roths JB, Murphy ED. Induction of various autoantibodies by mutant gene lpr in several strains of mice. J Immunol. 1984;133(1):227-233.

19. Wang Y, Nose M, Kamoto T, Nishimura M, Hiai H. Host modifier genes affect mouse autoimmunity induced by the lpr gene. Am J Pathol. 1997; 151(6):1791-1798.

\title{
A stimulating way to improve $T$ cell responses to poxvirus-vectored vaccines
}

\author{
Stuart N. Isaacs
}

University of Pennsylvania School of Medicine and Philadelphia VA Medical Center, Philadelphia, Pennsylvania, USA.

\begin{abstract}
Vaccines remain one of the most cost-effective public health measures. Despite ongoing efforts, protective vaccines against cancer and many infectious diseases, including malaria, tuberculosis, and HIV/AIDS, are still not in hand. Most investigators believe that to succeed against these difficult targets, vaccines that generate potent $T$ cell responses are needed. In this issue of the JCI, Salek-Ardakani et al. show how the relative virulence of a virus/vaccine vector affects the memory $\mathrm{CD8}^{+} \mathrm{T}$ cells generated and how the response may be enhanced. The work has important implications for the development of future vaccines that aim to trigger $\mathrm{CD8}^{+} \mathrm{T}$ cell responses.
\end{abstract}

Disease prevention is the key to public health. One of the most cost-effective ways to prevent disease is through vaccination, an approach that has enabled successful control of many infectious diseases that were once common, including measles, diphtheria, and pertussis (whooping cough). In addition, a protective vaccine was key to the global eradication of smallpox, and it is believed that vaccinations may soon relegate polio to a historical memory. However, many infectious diseases, including malaria, tuberculosis, and HIV/AIDS, have proven to be formidable foes; despite intensive research efforts, we still do not have efficacious protective vaccines. Most believe that to be successful, such vaccines - as well as vaccines that protect against cancer - will need to generate potent $\mathrm{T}$ cell responses.

Poxviruses are members of a large family of DNA viruses that share a long his-

Conflict of interest: The author has declared that no conflict of interest exists.

Citation for this article: J Clin Invest. 2011; 121(1):19-21. doi:10.1172/JCI45726. tory with vaccination. Cowpox virus was the active agent in Jenner's pioneering approaches to preventing smallpox in the 18 th century and is closely related to vaccinia virus, which was used as the vaccine that helped eradicate smallpox in the latter half of the 20th century. Remarkably, vaccinia virus remains one of the leading vector candidates to be used in the development of vaccines against challenging infectious diseases and cancers, largely as a result of the promise seen in the earliest studies that showed its utility as an expression vector $(1,2)$ and its ability to prime $\mathrm{T}$ cell responses to expressed foreign antigens (3). However, the laboratory strains of vaccinia virus, and even the old conventional human smallpox vaccines, are likely not ideal vaccine vectors from a safety perspective, especially in current times, when greater numbers of immunocompromised people are likely to be vaccinated or inadvertently exposed; even in normal individuals, what is considered an acceptable risk/benefit ratio has shifted. This has led to the pursuit of more attenuated and replication-incompetent poxvirus-based vaccine vectors that have significantly improved safety profiles. A recent achievement that highlights the promise of poxvirus-based vaccines is the somewhat unexpected results of the Thai HIV vaccine trial (4). In that study, vaccination with a recombinant Canarypox virus vector expressing three HIV proteins along with boosts of HIV envelope proteins showed modest protection from HIV acquisition. While some worry about the reproducibility of the effect found, others are clearly encouraged by the results and are seeking to understand and improve upon the immune responses generated by the vaccination strategy. Since vaccineinduced sterilizing immunity (defined as antibody and innate immune responses that prevent actual infection) is a high bar to reach against the problematic organisms that cause malaria, tuberculosis, and HIV/AIDS, future vaccines will need to generate potent antibody and cytotoxic $T$ cell responses.

In this issue of the JCI, Salek-Ardakani et al. show how the relative virulence of a virus/vaccine affects the memory $\mathrm{CD}^{+}$ $\mathrm{T}$ cells that are generated and how one might manipulate costimulatory molecules to enhance the $\mathrm{CD} 8^{+} \mathrm{T}$ cell response (5). The work has important implications for development of future vaccines that aim to target the $\mathrm{CD}^{+} \mathrm{T}$ cell response and the stimulatory molecules that might be candidates to generate such responses. 


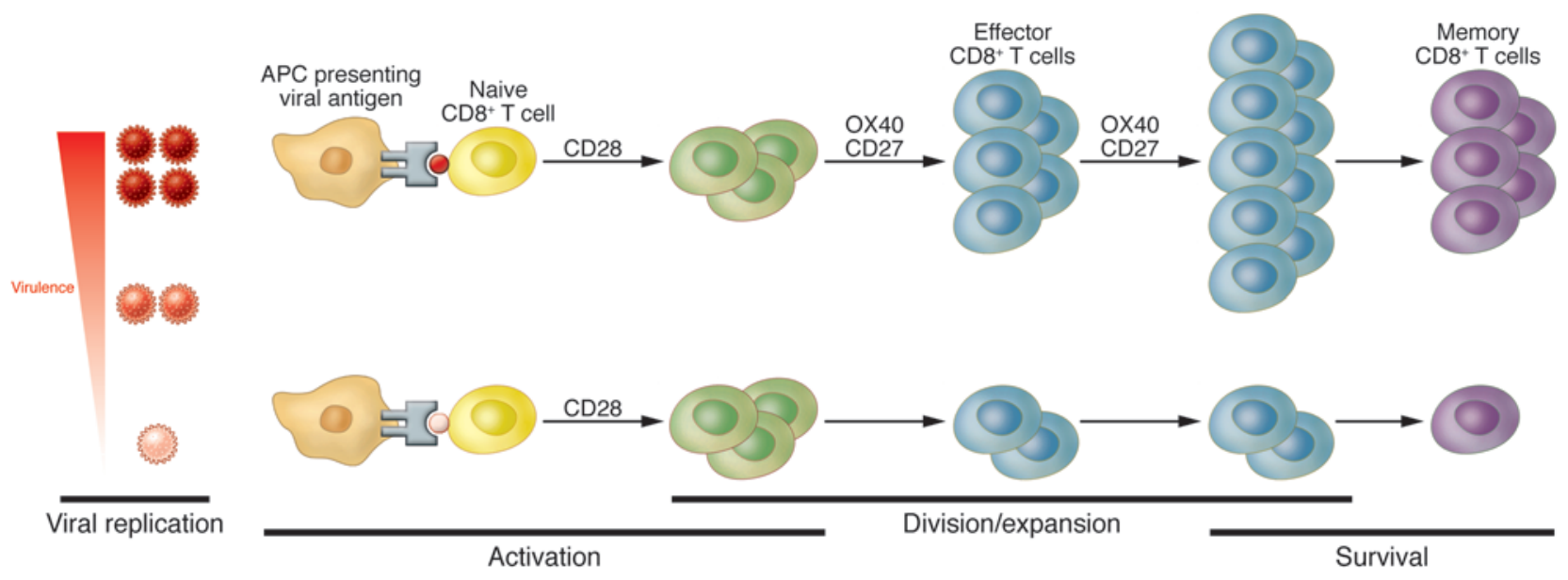

Figure 1

Viral virulence results in enhanced $C D 8^{+} \mathrm{T}$ cell memory responses. Compared with vaccination with a more attenuated virus (bottom), vaccination with a more virulent virus (top) causes stimulation of OX40 and CD27, which results in larger pools of effector and memory CD8 ${ }^{+} \mathrm{T}$ cells that can protect from subsequent lethal challenge.

\section{T cell responses to poxvirus vaccines}

Over the past two decades, there has been an increasing number of publications of human clinical studies with poxviruses as vaccine vectors against cancer and infectious agents, which indicates that basic poxvirus research has made a transition to clinical investigations. Clearly, vaccination with vaccinia virus generates long-lasting $\mathrm{T}$ cell responses to poxvirus antigens. For example, vaccinia virus-specific $\mathrm{CD}^{+}$and $\mathrm{CD}^{+} \mathrm{T}$ cells can be detected 6-8 decades after a single smallpox vaccination (6). In fact, the long-lived humoral and cellular immunity generated by the conventional smallpox vaccines create an additional challenge when using orthopoxviruses (i.e., poxviruses in the same genus as vaccinia virus) as vaccine vectors to immunize against other diseases: preexisting immunity to the vector dampens immune responses, especially when using a standard dose of replication-competent vaccinia virus-based vaccine (e.g., ref. 7). Because of the rare but significant complications that can occur with conventional smallpox vaccines, such as progressive vaccinia in immunocompromised hosts or eczema vaccinatum in some patients with common skin diseases, more attenuated poxviruses vectors have been developed. However, finding the perfect balance between safety and immunogenicity is challenging. For example, modified vaccinia Ankara (MVA), which is highly attenuated and does not generate infectious progeny in most mammalian cells, has been a lead candidate vector; however, human immune responses to foreign proteins encoded by MVA have been inconsistent. In small phase 1 human trials using an identical recombinant MVA encoding HIV proteins, one trial of 9 subjects revealed that approximately $90 \%$ developed $\mathrm{CD}^{+} \mathrm{T}$ cell responses after two intradermal vaccinations with $5 \times 10^{7}$ virus units (8), whereas another trial of 8 subjects reported that none of the subjects developed measurable $T$ cell responses after two intradermal vaccinations with $1 \times 10^{8}$ virus units (9). While these studies used different assays to define vaccine-induced $\mathrm{T}$ cell responses, it is clear that improving vaccine-induced $\mathrm{CD}^{+} \mathrm{T}$ cell responses in humans is needed.

\section{Enhancing T cell responses}

Although it might seem obvious that a more virulent virus results in more viral replication, which leads to better immune responses than those induced with attenuated viruses, Salek-Ardakani et al. asked whether there was a specific molecular mechanism engaged by the former but not the latter that could be harnessed to boost immunity generated by attenuated (and thus presumably safer) virus vaccines (5). Using a panel of vaccinia viruses with various levels of virulence in mice, the authors provide interesting data on how the relative virulence of a virus/vaccine affects the memory $\mathrm{CD}^{+} \mathrm{T}$ cells generated. When mice were infected with one of the more virulent viruses (i.e., laboratory vaccinia virus strain Western Reserve), it replicated longer and resulted in a larger pool of initially primed $\mathrm{CD}^{+} \mathrm{T}$ cells, and subsequently a larger memory $\mathrm{CD}^{+} \mathrm{T}$ cell pool, than did a less virulent virus (e.g., human smallpox vaccine Lister). To show the importance of the $\mathrm{CD}^{+} \mathrm{T}$ cell responses, genetically manipulated mice that lacked the ability to generate humoral immunity and thus relied exclusively on the $\mathrm{CD}^{+} \mathrm{T}$ cell response were shown to be protected from lethal vaccinia virus challenge only when vaccinated with the more virulent vaccine. The memory $\mathrm{CD}^{+} \mathrm{T}$ cells from mice vaccinated with the virulent vaccine were also the only ones that conferred protection against lethal challenge when passively transferred to naive mice prior to challenge.

Based on their prior study, in which they found that OX40 (also known as CD134) stimulation was important in determining the magnitude of antivaccinia $\mathrm{CD}^{+} \mathrm{T}$ cell responses (10), Salek-Ardakani et al. focused on this and other costimulatory molecules to define a mechanism of the improved memory $\mathrm{CD}^{+} \mathrm{T}$ cell responses observed with more virulent vaccinia viruses. They examined the role of a group of costimulatory molecules present on $\mathrm{CD}^{+} \mathrm{T}$ cells: OX40, CD27, and CD28. Experimentally, the authors found that in $O x 40^{-/-}$mice, the memory $\mathrm{CD}^{+} \mathrm{T}$ cell pool was greatly diminished only in animals infected with the most virulent vaccinia virus, whereas the memory pools generated after administration of attenuated vaccines remained relatively low and unchanged (5). Similar results were generated in $C d 27^{-1-}$ mice. For the panel of viruses with varying virulence, the $\mathrm{Cd} 28^{-/-}$mice gave memory $\mathrm{CD}^{+} \mathrm{T}$ cell responses similar to those seen in infected 
wild-type mice (albeit with slightly different kinetics), which indicated that for the panel of vaccinia viruses tested, there was a CD28-dependent phase of $\mathrm{CD}^{+} \mathrm{T}$ cell priming followed by a CD28-independent phase. Furthermore, by blocking CD27 in $\mathrm{Cd} 28^{-1-}$ mice, the authors found that CD27 was needed for normal CD28-independent priming of $\mathrm{CD}^{+}$cells. Based on these results, they proposed the model illustrated in Figure 1. As additional support for the role of OX40 in the development of a robust memory $\mathrm{CD}^{+} \mathrm{T}$ cell response, they used an antibody to stimulate OX40 and showed that this enhanced $\mathrm{CD}^{+} \mathrm{T}$ cell responses in mice infected with more and less virulent vaccinia viruses. Furthermore, the virus with lower virulence (which previously did not protect against lethal challenge), when used together with OX40 costimulation, generated $\mathrm{CD}^{+} \mathrm{T}$ cell responses that protected mice from lethal vaccinia virus challenge.

\section{Route of vaccination}

Another recurrent issue observed by SalekArdakani et al. was that the route of vaccina virus vaccination had an impact on the quality of the immune responses generated (5). Their work described above focused on intraperitoneal vaccination. When the authors vaccinated mice by scarification with the less virulent vaccinia virus human smallpox vaccine strain Lister, they found that its replication, resulting $\mathrm{CD}^{+} \mathrm{T}$ cell responses (dependent upon OX40 and CD27, as well as CD28), and ability to protect genetically altered mice from lethal challenge in a $\mathrm{CD}^{+} \mathrm{T}$ cell-dependent fashion was similar to scarification with the more virulent strain of vaccinia virus. The fact that scarification leads to enhanced immune responses has been a recurring finding with vaccinia virus vaccines (11-13) and likely points to the special role the skin plays in initiating immune responses. Interestingly, the enhanced immune responses - especially $\mathrm{T}$ cell responses - are not just seen with replicating vaccinia virus in the skin, but also with replication-incompetent vaccinia virus vaccine vectors such as MVA. The use of the skin as a site for vaccine delivery, and its ability to generate improved immune responses, is being examined for other vaccine targets (e.g., ref. 14).

\section{Questions and future challenges}

While the work by Salek-Ardakani et al. (5) is intriguing and provides insights into a molecular mechanism of how vaccinia viruses with varying degrees of attenuation induce different levels of $\mathrm{CD}^{+} \mathrm{T}$ cell responses, and how one might use the information to enhance responses, numerous important questions remain. Will the molecular mechanism of enhanced $\mathrm{CD}^{+} \mathrm{T}$ cell responses found with vaccinia virus infections be the same for other vaccines and/or infectious agents? How translatable will the findings and interventions be from mice to humans? Can a similar intervention of OX40/CD28 stimulation enhance immune responses in subjects whose preexisting poxvirus immunity dampens vector replication and leads to poorer immune responses? Although there is published evidence that OX40 engagement results in better $\mathrm{T}$ cell responses generated by replicationincompetent poxvirus vaccines (15), is the magnitude of this response at a level that can protect from lethal challenge? If one approach to provide costimulatory activity is to express specific ligands from a replication-competent recombinant vaccinia virus vector, one must proceed judiciously. When investigators were interested in enhancing antibody responses during the early development of a poxvirus-based immunocontraceptive vaccine to be used in the wild to control rabbit populations in Australia, they first constructed an ectromelia (mousepox) virus that expressed IL-4 (16). Not only was the resulting virus more virulent in mouse strains that were usually resistant to ectromelia virus, but it also resulted in mortality in mice previously vaccinated with a normally protective vaccine.

The potential use of poxvirus-based vaccine vectors against infectious disease and cancer targets continues to show great promise. The work by Salek-Ardakani et al. (5) adds to an ever-expanding body of literature that provides a molecular understanding of adaptive immune responses to the virus as well as insights into how such knowledge can be used to enhance immune responses to poxvirusbased vaccines.

\section{Acknowledgments}

The author is supported in part by Public Health Service grants U01-AI077913, U01AI066333, and U54-AI057168 (Middle Atlantic Regional Center of Excellence in Biodefense and Emerging Infectious Diseases) from the National Institute of Allergy and Infectious Disease.
Address correspondence to: Stuart N. Isaacs, University of Pennsylvania School of Medicine, Department of Medicine, Division of Infectious Diseases, 502 Johnson Pavilion, Philadelphia, Pennsylvania 19104-6073, USA. Phone: 215.662.2150; Fax: 215.349.5111; E-mail: isaacs@mail. med.upenn.edu.

1. Panicali D, Paoletti E. Construction of poxviruses as cloning vectors: insertion of the thymidine kinase gene from herpes simplex virus into the DNA of infectious vaccinia virus. Proc Natl Acad Sci US A. 1982;79(16):4927-4931.

2. Mackett M, Smith GL, Moss B. Vaccinia virus: a selectable eukaryotic cloning and expression vector. Proc Natl Acad Sci U S A. 1982;79(23):7415-7419.

3. Yewdell JW, Bennink JR, Smith GL, Moss B. Influenza A virus nucleoprotein is a major target for cross-reactive anti-influenza virus cytotoxic T lymphocytes. Proc Natl Acad Sci U S A. 1985; 82(6):1785-1789.

4. Rerks-Ngarm S, et al. Vaccination with ALVAC and AIDSVAX to prevent HIV-1 infection in Thailand. NEngl J Med. 2009;361(23):2209-2220.

5. Salek-Ardakani S, et al. The TNFR family members OX40 and CD27 link viral virulence to protective $\mathrm{T}$ cell vaccines in mice. J Clin Invest. 2011; 121(1):296-307.

6. Hammarlund E, et al. Duration of antiviral immunity after smallpox vaccination. Nat Med. 2003; 9(9):1131-1137.

7. Cooney EL, et al. Safety of and immunological response to a recombinant vaccinia virus vaccine expressing HIV envelope glycoprotein. Lancet. 1991;337(8741):567-572.

8. Mwau M, et al. A human immunodeficiency virus 1 (HIV-1) clade A vaccine in clinical trials: stimulation of HIV-specific T-cell responses by DNA and recombinant modified vaccinia virus Ankara (MVA) vaccines in humans. J Gen Virol. 2004; 85(pt 4):911-919.

9. Goonetilleke $\mathrm{N}$, et al. Induction of multifunctional human immunodeficiency virus type 1 (HIV-1)specific $\mathrm{T}$ cells capable of proliferation in healthy subjects by using a prime-boost regimen of DNAand modified vaccinia virus Ankara-vectored vaccines expressing HIV-1 Gag coupled to CD8+ T-cell epitopes. J Virol. 2006;80(10):4717-4728.

10. Salek-Ardakani S, Moutaftsi M, Crotty S, Sette A, Croft M. OX40 drives protective vaccinia virus-specific CD8 T cells. J Immunol. 2008;181(11):7969-7976.

11. McClain DJ, et al. Immunologic responses to vaccinia vaccines administered by different parenteral routes. J Infect Dis. 1997;175(4):756-763.

12. Liu L, Zhong Q, Tian T, Dubin K, Athale SK, Kupper TS. Epidermal injury and infection during poxvirus immunization is crucial for the generation of highly protective T cell-mediated immunity. Nat Med. 2010;16(2):224-227.

13. Wilck MB, et al. Safety and immunogenicity of modified vaccinia Ankara (ACAM3000): effect of dose and route of administration. J Infect Dis. 2010;201(9):1361-1370.

14. Sullivan SP, et al. Dissolving polymer microneedle patches for influenza vaccination. Nat Med. 2010;16(8):915-920.

15. Liu J, Ngai N, Stone GW, Yue FY, Ostrowski MA. The adjuvancy of OX40 ligand (CD252) on an HIV-1 canarypox vaccine. Vaccine. 2009;27(37):5077-5084.

16. Jackson RJ, Ramsay AJ, Christensen CD, Beaton S, Hall DF, Ramshaw IA. Expression of mouse interleukin-4 by a recombinant ectromelia virus suppresses cytolytic lymphocyte responses and overcomes genetic resistance to mousepox. J Virol. 2001;75(3):1205-1210. 


\title{
BCR-ABL kinase is dead; long live the CML stem cell
}

\author{
Alexander Perl and Martin Carroll \\ Division of Hematology and Oncology, University of Pennsylvania, Philadelphia, Pennsylvania, USA.
}

\begin{abstract}
Chronic myeloid leukemia (CML) is a hematopoietic disease characterized by expansion of myeloid blood cells. It is caused by the $t(9 ; 22)$ chromosomal translocation that results in the expression of the fusion tyrosine kinase BCR-ABL. Tyrosine kinase inhibitor (TKI) therapy has led to longterm remissions, but patients remain $B C R-A B L^{+}$. There is agreement that TKIs do not kill CML stem cells; however, it is controversial whether this is because of a lack of BCR-ABL kinase inhibition in CML stem cells or because CML stem cells do not require BCR-ABL for survival. In this issue of the JCI, Corbin and colleagues provide definitive evidence that $B C R-A B L$ is kinase active in CML stem cells and that TKIs inhibit this kinase activity without affecting CML stem cell survival. Rather, CML stem cells revert to a normal dependence on cytokines for survival and proliferation. These results demonstrate that the CML stem cell is not BCR-ABL addicted and have important implications for developing curative therapeutic approaches to CML.
\end{abstract}

Chronic myeloid leukemia (CML) is unique among human malignancies in its $1: 1$ association with the $B C R-A B L$ oncogene, which is generated by the hallmark Philadelphia chromosome $(\mathrm{Ph})$ that results from a $(9 ; 22)(\mathrm{q} 34 ; \mathrm{q} 11)$ reciprocal translocation that juxtaposes the Abelson murine leukemia viral oncogene homolog 1 $(A B L)$ gene normally located on chromosome 9 with the breakpoint cluster region $(B C R)$ gene on chromosome 22. Similarly, it is unique as the poster child for targeted therapeutics, in that the ABL kinase inhibitor imatinib has been spectacularly successful at durable restoration of $\mathrm{Ph}^{-}$ hematopoiesis and control of progression from CML chronic phase to blast crisis. Recent results from the earliest large trial of imatinib in North America have demonstrated a nearly $90 \%$ survival rate after 8 years of follow-up $(1,2)$. Despite this clinical success, BCR-ABL-containing hematopoietic cells fail to be fully eradicated in the vast majority of patients (3). As assessed by quantitative RT-PCR, $B C R-A B L$ transcripts in the blood and/or bone marrow remain measurable and generally stable for years during therapy (3). Importantly, the residual clones are fully capable of restoring leukemic disease, as noted by

Conflict of interest: $\mathrm{M}$. Carroll has received research support from Sanofi-Aventis Corp.

Citation for this article: J Clin Invest. 2011; 121(1):22-25. doi:10.1172/JCI43605. expansion of the malignant clone in most patients who discontinue imatinib therapy. Although it has been recently reported that a sustained, leukemia-free state can be maintained in rare individuals after controlled imatinib discontinuation, $\mathrm{CML}$ recurrence generally occurs even among patients who previously achieved RT-PCR negative tests for several years $(4,5)$. Thus, lifelong daily tyrosine kinase inhibitor (TKI) therapy is currently recommended for all patients newly diagnosed with CML. This approach incurs a substantial financial cost, is potentially limited in efficacy by patient compliance, and can be associated with severe side effects and/or secondary medical complications in some patients $(6,7)$. Hence, the scientific community has embarked on detailed, mechanistic studies of imatinib resistance (i.e., therapeutic failure) and persistence of BCR-ABL-containing hematopoietic cells. In this issue of the JCI, Corbin and colleagues provide new insights into this problem (8).

\section{Imatinib resistance is distinct from BCR-ABL persistence}

Importantly, the primary reason for early therapeutic failure of imatinib appears to differ from the probable causes of molecular persistence. Most patients whose leukemia initially responds to imatinib but then soon become resistant show emergence of secondary $\mathrm{Ph}^{+}$clones bearing mutations in BCR-ABL itself
$(9,10)$. These mutations impair the ability of imatinib to bind to, and thus inhibit, the enzymatic activity of the BCR-ABL kinase. This necessitates therapy with second-generation BCR-ABL inhibitors (e.g., dasatinib and nilotinib) that show more potent activity against native BCR-ABL as well as many of the described imatinib resistance mutations (11-13). Interestingly, low levels of these imatinib-resistant subclones can be demonstrated to precede imatinib therapy $(10,14)$. Thus, they likely represent clonal diversity of $\mathrm{Ph}^{+}$hematopoiesis at the time of disease diagnosis; and, upon clearance of the major $\mathrm{Ph}^{+}$clone during imatinib therapy, the imatinib-resistant minor clone now exhibits a relative growth advantage and replaces normal hematopoiesis (15). As would be predicted by this model, the outgrowth of imatinib-binding resistance mutations in BCR-ABL occurs early after the initiation of therapy, and their incidence actually appears to decrease with prolonged TKI therapy (16). Thus, imatinib resistance mutations do not explain the common finding of stable persistence of BCR-ABL ${ }^{+}$cells during years of imatinib therapy, raising the question of whether molecular persistence is BCR-ABL dependent or independent and whether its natural history is sinister or benign.

\section{The CML stem cell and BCR-ABL}

This question has led to a series of investigations of the CML stem cell. It is generally agreed that the leukemia-initiating cell in CML is a BCR-ABL ${ }^{+} \mathrm{HSC}$ that, per current knowledge, is immmunophenotypically indistinguishable from normal HSCs and has the phenotype $\mathrm{Lin}^{-} \mathrm{CD} 34^{+} \mathrm{CD} 38^{-}$. The Holyoake lab was the first to demonstrate that $\mathrm{CD} 34^{+}$cells from the bone marrow of patients with CML are able to survive in the presence of imatinib and other ABL kinase inhibitors (17). Others have confirmed this observation, but there is substantial controversy as to whether TKIs actually inhibit BCR-ABL kinase in the quiescent stem cell fraction of CML 